\title{
Entrevista a Patricio Meller: la labor del Consejo Asesor Presidencial para la Equidad y el Trabajo
}

Este comentario, junto a los documentos que analiza, está disponible en www.anuariocdh.uchile.cl

Por Claudia Sarmiento*

Patricio Meller es chileno, Ingeniero Civil de la Universidad de Chile, Máster en Ciencias (Ingeniería) y Doctor en Economía de la Universidad de California, Berkeley. Profesor Titular de Ingeniería Industrial de la Universidad de Chile e Investigador de CIEPLAN. Fue Presidente del Consejo Asesor Presidencial sobre Trabajo y Equidad (2007-2008), Director de CODELCO (2000-2006), Director Departamento de Ingeniería Industrial, Director Académico Magíster en Gestión para la Globalización (Universidad de Chile). Profesor Visitante Universidad de Notre Dame y Universidad de Boston, Investigador Invitado National Bureau of Economic Research (NBER) de Nueva York, Profesor Jornada Completa Instituto de Economía, Universidad Católica (1970-1974). Autor y editor de 15 libros, en español, inglés y francés. Artículos publicados: 66 en revistas y libros especializados en economía. Consultor internacional WIDER, BID, OECD, Banco Mundial, UNCTAD.

Claudia Sarmiento: El trabajo del Consejo Asesor Presidencial del Trabajo y la Equidad debía abordar las distintas dimensiones del derecho a la igualdad y las situaciones de discriminación, particularmente las causas y estructuras que refuerzan la discriminación y la exclusión en las políticas económicas, en la legislación laboral y en las políticas públicas en el mundo del trabajo.

Después de leer el informe del Consejo, me interesan especialmente dos puntos.

El primero de ellos guarda relación con el funcionamiento del Consejo. ¿Cómo alcanzaron los consensos a través de los cuales se construye el informe? Mi impresión es que parten de la noción de igualdad de oportunidades como un marco teórico de las políticas que después proponen. ¿Por qué escogieron el concepto de "igualdad de oportunidades"? ¿Hubo algún cuestionamiento respecto a los modelos económicos y la generación del trabajo?

El segundo punto tiene que ver con la puesta en práctica de las medidas sugeridas por el Consejo. ¿Cuáles estima serán las principales dificultades de la implementación de las propuestas de la Comisión de Equidad? ¿Cuál es el plazo en que se proyectó la implementación de las propuestas? ¿Qué resultados se espera obtener en la hipótesis de que estas medidas se implementen?

Patricio Meller: Las dificultades que tuvo este Consejo, respecto a otros consejos asesores presidenciales, residieron en el marco del problema central que debió abordar: la equidad. Al

\footnotetext{
Licenciada en Ciencias Jurídicas y Sociales de la Universidad de Chile (distinción máxima), de la Facultad de Derecho de la Universidad de Chile. Actualmente se desempeña como investigadora del Programa de Mujeres y Derechos Humanos, del Centro de Derechos Humanos $(\mathrm{CDH})$ de la misma Casa de Estudios.

csarmien@derecho.uchile.cl
} 
contrastarlo, por ejemplo, con el Consejo de Reforma Previsional, este último tuvo términos de referencia bastante enmarcados, porque existe un sistema de previsión social implementado en 1981 y que ha estado en funcionamiento durante los últimos veinticinco años, y sobre la marcha del mismo se han detectado los problemas que tiene. La discusión sobre este régimen podía acotarse a cómo revisar y corregir las deficiencias que tiene este sistema de capitalización individual de previsión social. El tema está definido y los problemas subyacentes son percibidos empíricamente, y ha habido propuestas para hacer más equitativo el sistema y para incluir a quienes están fuera de él.

El otro elemento importante respecto a la generación de consenso, y siguiendo con el ejemplo de la Comisión de Reforma Previsional, es que todas las personas que conformaron el equipo de trabajo de éste (alrededor de dieciséis personas) eran técnicos especialistas en el tema, por lo que hubo cierta afinidad innata, y un lenguaje y un know how comunes.

Comienzo con estos ejemplos, para destacar el tipo de desventajas con las que parte el Consejo de Equidad.

A lo anterior deben añadirse los factores del contexto en que la Presidenta llama a la formación de este Consejo Asesor. El primero de ellos son los conflictos laborales que se habían desencadenado, los cuales crean ambientes de tensión de alta intensidad dentro del país. El segundo factor es que, antes de que se produjera un desenlace grave de los conflictos laborales, entró al escenario la Iglesia, y particularmente Monseñor Goic, planteando que es necesario establecer un salario equitativo, proponiendo además el monto de \$250.000 mensuales. Esta no es la primera vez que la Iglesia evidencia que éste no es un país equitativo y que hay que tomar medidas que traten de corregir este nivel de inequidad. Dado que esto se planteó en una situación particular, se generaron reacciones inmediatas en toda la sociedad. La propuesta del Obispo Goic se entendió como algo que la sociedad quiere y percibe como justo. Eso es en cuanto al contexto.

El Consejo, entonces, se constituyó con un grupo de personas totalmente heterogéneo, dentro del cual había tanto académicos como políticos; consejeros con sensibilidad empresarial y otros con sensibilidad sindical, es decir, una combinación compleja. Dado que la propuesta nació desde la Iglesia, había tres representantes de ella (dos católicos y un evangélico), junto a gente laica, agnóstica o atea, todos los cuales conformaron una combinación extraña. Había gente de distintas disciplinas: economistas, abogados laboralistas, entre otros. Y como última combinación de personas, tuvimos simpatizantes de la Concertación, simpatizantes de la Alianza e independientes. Todos ellos sumaban cuarenta y ocho personas.

\section{CS: Un grupo representativo...}

PM: Dadas la diversa composición del grupo de personas y las discusiones que se generaron, podría decirse que el Consejo representaba la sociedad chilena.

Una de las primeras discusiones que tuvo lugar en la primera reunión, tenía que ver justamente con el otro problema de fondo de la Equidad: cómo se define, de qué estamos hablando cuando hablamos de equidad, cuál es el problema que nos convoca. Se generaron dos visiones distintas sobre lo que es la equidad y cómo aproximarse a resolver el problema de la inequidad.

La primera visión, sostenida por un grueso de los consejeros en el principio del trabajo, suponía percibir el problema como la elaboración de propuestas destinadas a hacer "algo" por los pobres.

La segunda visión, alojada en los representantes de los trabajadores, planteaba que el problema de la equidad está en las relaciones laborales, entre trabajadores y empresarios, y que las propuestas debían estar dirigidas a mejorar la situación de los trabajadores. 
Son dos miradas bastante diferentes, que corresponden a la combinación diversa de los integrantes que conformaban el Consejo.

¿Cómo se zanjó esto? Y también, operativamente, ¿cómo trabajar con cuarenta y ocho personas? El número cuarenta y ocho tiene una virtud matemática bastante importante: es divisible por varios números, entre ellos, el tres, por lo que constituimos tres subcomisiones de dieciséis personas cada una. Y no es accidental que sean dieciséis. Conversé largamente con Mario Marcel quien era el presidente del Consejo Asesor de la Reforma Previsional, y me interioricé de la mecánica con la que operaron. Esto ayudó a que el Consejo de Equidad tuviera resuelto desde el segundo meeting los problemas logísticos y de funcionamiento.

Las subcomisiones resolvieron el cómo abordábamos el problema de equidad. Una de ellas se dedicó a tratar las políticas para la equidad de los grupos de menores ingresos. Otra de las subcomisiones, en relación a algo que anticipamos, trabajó sobre los temas del mercado de trabajo a través de políticas laborales. La tercera subcomisión respondió directamente a lo que plantearon los trabajadores: cómo hacer más simétrico el poder de negociación colectiva entre trabajadores y empresarios, dentro de la empresa, y cómo enfrentar el punto de la negociación laboral.

Esa fue la forma pragmática que utilizamos para enfrentar el problema.

Desde la primera reunión, puse énfasis en que la sociedad chilena no estaba interesada en nuevos diagnósticos sobre el problema, y que lo que esperaba de este Consejo Asesor Presidencial no era sino propuestas, y es por eso que las subcomisiones debían elaborar propuestas concretas en relación directa con las áreas definidas, dentro de ciertos plazos. Si nos fijamos en el contenido informe, el $9 \%$ es diagnóstico y el restante $91 \%$ son propuestas. Lo que hicimos fue mirar lo que se ha hecho en Chile y en el extranjero, y determinar qué nos servía y qué podíamos plantear para el Chile de 2008 en adelante.

Dicho todo esto, parecía importante decantar, a nivel conceptual y revisando la literarura especializada, qué se entiende por equidad. Cuando hablamos de equidad, ¿de qué estamos hablando? Esto está consignado en forma muy esquemática en el informe. Es importante tener presente que equidad tiene cuatro dimensiones distintas.

La primera dimensión es la de inclusión-exclusión, en la cual está implícito que hay algunos sujetos que están excluidos de la sociedad, de la economía y del mercado, y que, de alguna forma, las políticas deben estar dirigidas a incluirlos. Estos sujetos son los pobres, los marginados, etc. y es preciso incluirlos por medio de políticas especiales. Deben ser valorados como seres humanos y no quedar eternamente marginados.

La segunda dimensión es la de inequidad o de desigualdad de oportunidades. La primera dimensión habla de casos de personas que están bajo un nivel determinado de pobreza y queremos establecer políticas que les permitan llegar a un nivel de subsistencia mínimo, pero esta segunda dimensión apunta a que personas que han partido en la vida desde lugares distintos, puedan tener las mismas oportunidades. El ejemplo tradicional es el de un niño o niña que nace en La Dehesa y otro que nace en La Pintana ${ }^{1}$. Desde su nacimiento se pueden anticipar sus trayectorias de vida; entonces surge la pregunta: ¿por qué algo tan accidental, como el lugar de nacimiento, condiciona el futuro de una persona? Cada uno sabe lo que hace con su vida, pero hay que tratar de darle a todos las mismas posibilidades. Entonces esta dimensión apunta a qué hacer con los niños al nacer, en la educación preescolar, básica y media, para que a los diecisiete años todos

1 La Dehesa y La Pintana representan, respectivamente, la comuna más rica y la más pobre de Santiago, la capital de Chile. 
queden más o menos en igualdad de oportunidades para decidir qué hacer de ahí en adelante. Y el tipo de política de esta segunda dimensión de la equidad es diferente al tipo de política que requiere la primera.

La tercera dimensión es la de movilidad social. El mejor ejemplo para entender de qué se ocupa esta dimensión es el siguiente: toda la sociedad chilena está dentro de un tren con distintos vagones de distinta clase (primera, segunda y tercera clase). Lo que ha pasado en los últimos veinte años en Chile es que la economía ha crecido más rápido y todas las clases sociales han aumentado su nivel de ingresos. No obstante, cuando se señala que hay poca movilidad social, se está diciendo que muy pocos pasan de un vagón a otro: de tercera clase a segunda clase, de segunda clase a primera clase. El tren puede ir más rápido, pero cada una de las clases permanece en su vagón. La estratificación social se mantiene constante a través del tiempo. Entonces, hay que generar otro tipo de políticas que permitan la movilidad social, distintas a las de las dimensiones de equidad antes mencionadas.

Y la cuarta dimensión tiene que ver con la vulnerabilidad social. En la sociedad chilena existen distintos tipos de familias, dentro de las cuales se encuentran las monoparentales (un solo jefe de hogar). Cuando el jefe de hogar (hombre o mujer) es despedido de su trabajo o se enferma, este hecho repercute fuertemente en toda la familia, pues condiciona o anula su acceso a la educación, salud, etc. Entonces allí hace falta otro tipo de políticas para disminuir los niveles de vulnerabilidad social.

Las tres subcomisiones se ocuparon de estas cuatro dimensiones de manera distinta. Por esto quise destacar lo difícil que fue trabajar sobre el tema de la equidad. La presión por ser pragmáticos y elaborar propuestas nos Ilevó a evitar filosofar especulativamente sobre cuál de estas dimensiones íbamos a privilegiar: más bien, debíamos poner el foco en cómo hacíamos operativas estas dimensiones y resolver si efectivamente la forma como estamos llevando a cabo la "igualdad de oportunidades" era la más adecuada, o la única.

\section{CS: ¿Las cuatro dimensiones de las que usted me habla nacieron de un consenso previo al trabajo de las subcomisiones?}

PM: No. Esto nació de un proceso de learning by doing donde se fueron estableciendo consensos a medida que se fue discutiendo. No es que todos estén de acuerdo con estas dimensiones, pero de a poco fuimos decantando, fuimos intercambiando opiniones, nos fuimos conociendo. De los cuarenta y ocho que estábamos en ese Consejo, yo conocía a menos de la mitad, así que tuvimos que desarrollar lazos de confianza con el resto, generar un lenguaje común poniéndonos de acuerdo sobre de qué íbamos a estar hablando y de qué no. Generamos una mecánica de funcionamiento semanal, en la que todo el Consejo estuvo dispuesto a reunirse todos los miércoles, todo el día. Todos los coordinadores de comisiones tuvieron que trabajar dos días al principio, y después aumentábamos un día cada mes. Los ejecutivos terminamos trabajando siete días a la semana. Todo ad honórem: nadie cobró un peso. La gente se involucró.

Muchos venían con ciertas ideas y prejuicios sobre un cúmulo de cosas, pero la mayoría estuvo dispuesta a escuchar cuáles eran las razones que daba la contraparte y hubo quienes finalmente cambiaron de opinión, dándose cuenta de que la discusión era bastante más compleja. Incluso los que no cambiaron de opinión, entendieron las razones del otro.

CS: Mi inquietud sobre el cómo se generaron los consensos y el marco teórico sobre la igualdad estaba motivada precisamente por la diversidad de los miembros y lo complejo que es hablar 
de equidad o de inequidad y de su significado. También porque tengo la sensación de que en Chile subsisten varios parámetros culturales que de una u otra forma inciden en esta discusión. Hasta hace poco tiempo, hablar en Chile de distintos tipos de familia era complicado, porque subyacía al discurso político una única concepción de familia, a la que además se le adjudicaba la condición de legítima. Ahora bien, el sustrato social y las prácticas diferían del discurso, pero éste incidía mucho en las políticas, particularmente en qué es lo que desea el Estado reconocer como legítimo.

Me imagino que en ese contexto, por ejemplo, debatir sobre cuál es el rol de la mujer en el tránsito del trabajo reproductivo no remunerado al trabajo formal remunerado, los incentivos que puede haber versus el rol que se le asigna en la maternidad, pueden haber causado dificultades o roces, o no haber estado en la representación de las cuatro dimensiones de equidad que mencionó.

PM: Sí. Pero hicimos algo sin anticipar su efecto: celebrar audiencias. Al principio nos preguntamos bastante "¿a quién representamos nosotros?", pues fuimos designados "a dedo". Había gente inscrita en partidos políticos. En mi caso, soy independiente, aunque simpatizante de la Concertación. Ninguno de nosotros fue elegido por representación popular y, por esa razón, las decisiones no las podíamos zanjar votando. Nada se votó. En algunas áreas, como se puede ver en el informe, hay dos o tres posturas distintas, y eso lo consignamos. Por eso, tomando como ejemplo la labor de otros Consejos, organizamos audiencias civiles con distintos grupos representativos de un amplio espectro de sectores sociales.

Establecimos un formato y una metodología. Les preguntábamos por definiciones de equidad y también sobre posibles soluciones. Todos los miércoles en la tarde destinábamos cuarenta y cinco minutos para las audiencias. Vinieron organizaciones de distinto tipo: comunitarias, de pobladores, asociaciones de trabajadores, asociaciones empresariales, partidos políticos, organizaciones no-gubernamentales, fundaciones, centros de consultores; nadie participó a título individual. Y todo esto fue hecho en la Moneda. El Consejo tenía mucho "bombo" al principio, y era cuestionado por la $\mathrm{CUT}^{2}$, aunque hubo dirigentes que nos transmitieron la sensibilidad de la CUT. Hubo tanta demanda por venir a estas audiencias, que tuvimos que extenderlas. Mucha gente pasó por las audiencias.

Uno de los temas tratados fue el rol de la mujer en la sociedad chilena, en torno a lo cual hubo dos posturas distintas, sobre todo en cuanto a lo que había que hacer para favorecer la igualdad de la mujer, teniendo en cuenta el dilema entre trabajo y familia que planteabas hace poco. También este aspecto está en nuestra encuesta de percepciones sobre equidad y mercado laboral.

Para académicos como yo, acostumbrados a estar frente a un escritorio, rodeados de libros y artículos y haciendo clases, fue ver pasar a Chile durante dos meses, y conocer los distintos problemas y visiones que había. Eso nos sensibilizó.

Concurrieron ante nosotros muchos dirigentes poblacionales, líderes de organizaciones no-gubernamentales, de iglesias, asociaciones de empleadas domésticas. A todos les exigíamos que hicieran una presentación por escrito, para que no improvisaran. La mayoría acompañó presentaciones en Power Point. La forma en que ellos hacían sus planteamientos era muchas veces más directa y más fundamental que la de nosotros, los académicos. En algunos casos, nosotros desarrollábamos en diez artículos lo que estos dirigentes decían en dos o tres frases.

2 Central Unitaria de Trabajadores. 
El hecho de que ellos expresaran su gratitud porque se les escuchara, porque por primera vez se les daba la voz, por estar por primera vez en la Moneda, cala hondo; porque en cuanto a equidad, mayormente pensábamos en el nivel económico o material, pero no tanto en lo que se necesitaba a nivel social.

CS: ¿Y se discutió esa dimensión social en la Comisión? La dimensión que usted rescata en este momento, el acceso al poder, el acceso a la vida pública, el poder incidir en los debates políticos, parece no tener una relación inmediata con el trabajo de las subcomisiones. Y eso también es parte, de una u otra forma, de la igualdad.

PM: Al respecto, después de revisar algunos modelos, se me ocurrió, desgraciadamente sin éxito, dejar un registro, que se podría haber Ilamado algo así como "La voz de los sin voz". El Gobierno ha hecho cosas similares, pero tienen muy poca difusión. A nosotros nos complicó, porque teníamos mucha presión por producir propuestas que después fueran transformadas en proyectos de ley y se volvieran instrumentos permanentes de solución de los problemas de equidad.

CS: Esa es una visión interesante acerca de cómo construir, porque deja de ver a las personas como las beneficiarias u objeto de políticas públicas, para ser un sujeto titular de derechos, que se hace parte de los procesos políticos. Estas audiencias, ¿piensa usted que le dieron al Consejo la legitimidad como "representante"?

PM: No. El objetivo funcional que tenían para nosotros las audiencias era sensibilizarnos respecto a cuáles son las propuestas que calan y cuáles son pura retórica, y qué era lo que teníamos que hacer. Las audiencias plasmaron en personas de carne y hueso el problema, según la voz de esa gente, para no irnos "por las ramas", como usualmente nos pasa a los académicos. Recordar esas voces, con frecuencia ayudó a que no nos dispersáramos y pudiéramos enfocarnos en el problema.

CS: Me interesa lo que se constata en el informe sobre igualdad de oportunidades en relación a la educación, especialmente en las etapas escolar y preescolar. Sé que hubo otro Consejo que trabajó paralelamente los temas de educación y no sé en qué medida ellos tocaron el tema de la equidad, o ustedes el de la equidad en la educación.

PM: No quisimos. No pudimos.

Cuando hablamos de equidad es un tema demasiado amplio que lo cubre todo. No tiene cotas. La única restricción que yo sentí fue, precisamente, la necesidad de no tocar los temas que ya estaban trabajando otras comisiones. Y justamente nos criticaron mucho porque hayamos dicho tan poco sobre educación. Pero ¿cómo íbamos a abarcar educación si había dos consejos separados para ello: uno para educación superior y otro para educación básica y media? Sin embargo, en un momento fue tanta la crítica que cuando fuimos al Parlamento me dijeron: "¿y usted no cree que la educación algo tenga que ver con el problema de equidad?". Entonces comprendimos el mensaje, y por eso una de las propuestas está específicamente enfocada a los niños de educación básica y media. Uno de los mayores problemas al respecto es cómo hacer para que los recursos les lleguen a los que les tiene que llegar. Dada esa situación, se nos ocurrió focalizar de la manera inversa, es decir, por exclusión. ¿A quién no queremos que le lleguen los recursos extras? No queremos que le lleguen al grupo más rico de este país, porque no los necesita. No queremos que les lleguen a los niños que están en colegios particulares pagados (totalmente privados). Por ello, excluimos a ese sector de nuestras propuestas y diseñamos un programa para tres mil quinientos colegios. 
¿Cuál es la crítica que se formula respecto de la $\mathrm{PSU}^{3}$ (y la anterior $\mathrm{PAA}^{4}$ )? Que compiten niños y niñas de colegios con distinto training, con distinta calidad de profesores e infraestructura. Nos preocupaba que alguien talentoso no tenga la oportunidad de ingresar a la Educación Superior. Por eso propusimos detectar los mejores estudiantes en todas partes, lo mejor en cada curso, en cada colegio, de $7^{\circ}$ básico a $4^{\circ}$ medio, para que tengan la posibilidad, independientemente de dónde estén, aunque sea en sectores rurales a miles de kilómetros de Santiago, de desarrollar al máximo su potencialidad. Esa es la lógica de ese programa.

CS: Me da la impresión, luego de leer el informe, de que estas políticas evidencian las falencias que hay en el sistema de meritocracia. Quiénes son los que Ilegan a las mejores universidades. ¿Eso se cuestionó de alguna forma?

PM: No. ¿Por qué establecimos este sistema? Según un estudio, un programa de capacitación preuniversitaria puede tener un impacto de mejorar en hasta cien puntos el rendimiento de un estudiante en la PSU. Entonces poder cursar o no un preuniversitario hace una gran diferencia en cuanto a si ingresas o no a la universidad. Lo que quisimos fue detectar a los estudiantes talentosos entre $7^{\circ}$ Básico y $3^{\circ}$ Medio, y pagarles un preuniversitario en $4^{\circ}$ Medio. Ahí es donde tratamos de hacer operativo este problema de que tú hablas. Si te fijas, en toda la discusión en los Consejos de Educación nunca hubo una propuesta para tratar de corregir esto. Y nosotros en el Consejo de Equidad cuantificamos y dimensionamos en dinero la propuesta: es la que está más detallada en cuanto a la implementación a través del tiempo. ¿Por qué así? Porque corregir el problema de fondo va a tardar, quizás, veinte años, y esta es una medida para lo inmediato. Queríamos hacer una propuesta que fuera más allá, pero los profesores nos frenaron, ya que nos podría traer más problemas que beneficios, y podría haber redundado en un cuestionamiento de todo el informe. Así que hicimos esta propuesta que llega a setenta mil niños de colegios municipales y particulares subvencionados.

\section{CS: Esta es una pregunta que ha estado en la palestra en el debate sobre educación ¿Abordaron el sistema de lucro y la descentralización en la educación particular subvencionada y municipal, respectivamente?}

PM: No, porque no es el tema de fondo. Hace pocos días tuvimos a un brillante experto inglés que habló sobre cómo ellos hicieron la reforma educacional en Inglaterra, y nos reveló que ese no es el problema de fondo. De hecho la Comisión de Educación estuvo discutiendo el tema equivocado.

\section{CS: Y el tema de fondo es...}

PM: Cómo se mejora la forma cómo se enseña. Lo que pasa dentro de la sala de clases. Todo pasa dentro de la sala de clases.

\section{CS: ¿Y ese es un tema ligado a la calidad de la docencia?}

PM: No, sino cómo se enseña. El problema existe no sólo en los colegios públicos, sino también en los colegios privados. Está en el arcaico sistema de enseñanza que tenemos. Y también ese arcaico sistema está en las universidades. Este es el problema. Entonces, lo que decía este profesor, es que hay que abrir la puerta de la sala de clases, y hacerla pública, que la gente vea cómo se enseña hoy, que entre gente a evaluar cómo los profesores están enseñando. Eso ni siquiera lo hacemos en las universidades. Ni siquiera en la Universidad de Chile, donde hay mucha resistencia

3 Prueba de Selección Universitaria.

4 Prueba de Aptitud Académica. 
a cambiar. Si ni siquiera lo hacemos en la universidad, ¿qué se espera para las instancias de educación previas?

Ahora estoy muy sensibilizado con este tema y creo que este es un asunto que cambiará prontamente. Un director de MBA inglés me dijo: "ven, entra a ver", y estuve en un par de clases donde justamente se enseñaba un par de tópicos que yo también enseño en la Universidad. Después el director me preguntó “¿y qué te pareció?", y yo le respondí "más o menos, porque usted ocupó treinta minutos en lo que yo me demoro tres horas y profundizo más, por lo que me pareció que fue muy superficial". Y la respuesta fue "¿y tú crees que los alumnos aprenden de los profesores? No, los alumnos no aprenden de sus profesores, sino de sus compañeros". "¿Pero cómo?", me pregunté yo. Después fui a otra sala de clases y me di cuenta de que él tenía toda la razón. El profesor era como un director de orquesta y hacía debatir a los alumnos. Eso sí, hay una diferencia en esos cursos: los alumnos eran de distintos países, del extranjero, y sólo el 25\% era local. La forma cómo pensaban los hindúes era muy distinta a la forma cómo pensaban los africanos, los de Europa Oriental, o los norteamericanos, o, incluso, los ingleses.

\section{CS: No eran alumnos pasivos, sino que la mayor parte del trabajo estaba en ellos...}

PM: Eran súper activos. Y, efectivamente, el profesor era genial, porque era como un director de orquesta, Ilevaba a la discusión y los hacía interactuar. Nuestros alumnos chilenos que estaban en ese programa, me decían: "Profe, primera vez que veo cómo piensa un chino, o cómo piensa un árabe, o cómo piensa un ucraniano, o cómo piensa un persa", porque piensan distinto.

\section{CS: Tienen diferentes respuestas para un mismo problema.}

PM: Claro. Y eso te hace pensar. Estos métodos de enseñanza tratan de abrir tu mente, no de que cites las cosas que dicta el profesor. Y eso ¿se discutió en los Consejos sobre Educación?

\section{CS: Mi impresión a priori es que no.}

PM: Y ese es el mayor problema.

\section{CS: Volviendo a la segunda pregunta que le formulé al empezar esta conversación ¿Cuáles son las perspectivas de implementación de las medidas que propone el informe del Consejo Trabajo y Equidad?}

PM: En el Consejo hubo gente de un amplio espectro político, que están o van a estar en los comités de programas de los distintos candidatos presidenciales, y saben lo que está en el informe. Gracias al sesgo academicista de los coordinadores que dirigíamos este proyecto, dejamos todo escrito. Esa gente sabe que hay propuestas aterrizadas para la sociedad chilena y las va a tomar y llevar en distinta dirección. Hay algunas propuestas que combinan, por ejemplo, la mejor política social norteamericana con la mejor política social latinoamericana, y nosotros sugerimos ciertos porcentajes de ponderación y otros elegirán otros porcentajes entre unas y otras políticas, pero las ideas están en el informe. ¿Cuánto demorarán en implementarlas? ¿Diez o veinte años? Al menos está la base de dónde empezar a discutir y no volver nuevamente a la hoja cero. Y dada la calidad de la gente que integró este Consejo, la seriedad con que está hecho el informe es conocida. No lo digo porque yo esté involucrado, sino porque enviamos el informe a organismos multilaterales, como el Banco Mundial y el Fondo Monetario Internacional, y sabemos que compite con las mejores publicaciones de los organismos multilaterales. La respuesta de estas instituciones fue "¿cómo podemos llevar esto al resto de los países latinoamericanos?". Las propuestas de esos organismos son hechas desde fuera, pero nuestro informe está hecho desde acá, Chile, desde un consenso social, desde una sensibilización por lo que está pasando acá, 
con distintas visiones. Y cuando tuvimos discrepancias, las dejamos consignadas, para que cuando se reinicie la discusión sobre estos temas, no se parta de cero, porque ya hay una base sobre la cual partir. Esto fue percibido por la gente del Gobierno, del Parlamento; en la Academia lo usan, los alumnos lo estudian, y va a permear las universidades. Los académicos tenemos el sesgo de que estas ideas importan, y estas son las que van a cambiar la sociedad.

Si me preguntas qué se ha implementado de esto hasta ahora, la respuesta sería "nada". Pero no importa, porque sé que lo que hicimos tiene proyección, porque hay ideas de fondo, según la gente, profesores y otros.

CS: Leí en el informe sobre el cambio de las premisas de las políticas sociales del siglo XX por las de las políticas sociales del siglo XXI, pasando de indicadores puramente cuantitativos a indicadores cualitativos. Eso es un cambio bastante significativo. ¿Es esa su visión personal o es una conclusión del tipo "learning by doing"? ¿Cómo se generó esto?

PM: Yo creo que fue una mezcla. Esto fue para mí una especie de programa de doctorado. Paré todo lo que estaba haciendo por meses; me dediqué a esto de lleno. Yo trabajo en CIEPLAN y también en Ingeniería Industrial, y los directores me dijeron que el trabajo en la Comisión era primera prioridad nacional, y que postergara lo demás. Pero aún después de terminar, no he podido parar.

Leí mucho de distintas partes, de las intervenciones de la gente, de distinta gente, de repente algunas cosas me hacían "click". Por ejemplo, el movimiento de los escolares pidiendo calidad, caló en mí muy adentro, y te das cuenta de que es un asunto que va más allá. Y uno se da cuenta de que ataca la forma cómo se ha hecho política hasta ahora. Es vital cambiar del paradigma cuantitativo a un paradigma cualitativo, en el que hay que definir los indicadores cualitativos. Esto es fácil criticarlo, y en algunas áreas se puede hacer mejor que en otras.

Hay mucha gente que todavía tiene la visión de cómo era Chile en 1990, particularmente dentro de la Concertación. Entonces las propuestas que dan están insertas en el pasado, cuando funcionaba bien el paradigma cuantitativo. Pero en la sociedad chilena del 2008, las exigencias son otras. La gente con otro nivel de ingresos exige, y cada vez va a exigir más, tal como pasa en los países desarrollados, donde ya no se requiere solamente que te atiendan, sino que te atiendan bien en los distintos servicios. Y también funciona al revés, cuando te relacionas con un empleado público ya no tienes temor a que por cualquier cosa no te atienda bien, sino que ahora él trabaja para ti. Yo viví mucho tiempo en Estados Unidos y eso lo tengo bien interiorizado, porque allí los ciudadanos decían "con mis impuestos se le paga su sueldo, así que atiéndame, y atiéndame bien". La gente que trabaja en el sector público lo siente así allí, pero aquí todavía no, aunque cada vez van a sentir una presión mayor. Esto comenzó con los escolares de colegios públicos, porque ellos lo percibieron en carne propia al ver cómo ellos quedaban en desventaja frente a los colegios privados. Y me llamó la atención lo que decían los dirigentes estudiantiles de los colegios privados: "yo no quiero competir en ventaja respecto al resto de jóvenes de mi generación de los colegios públicos. Yo quiero competir en igualdad de condiciones". Eso te lo dice un joven de 17 años de colegio privado, un "privilegiado". Entonces, hay que mejorar la calidad.

\section{CS: Hay una convicción democrática en las generaciones jóvenes...}

PM: Claro, y se ha permeado en cómo piensan los jóvenes, y cómo exigen. Pero también ha pasado a otras áreas, como la Salud, porque ipor qué la salud en un hospital público debería ser distinta a la de una clínica privada? Eso está calando fuerte en la gente. Por ejemplo, en un padre cuyo hijo tiene que operarse, tiene que vivir la espera en el sistema público o los costos de una clínica privada. Esto no pasa en los países desarrollados como en los países europeos o Canadá, aunque sí en Estados Unidos. 
Tú ves lo que pasó con las viviendas populares, que tienen que cubrirlas de la lluvia poniéndole plástico. ¿Por qué? ¿Cómo puede ser esto? Y cada vez que llueve la gente de bajos recursos pierde todos los activos que tiene, eso no puede ser. Eso está produciendo ruido.

CS: Para ahora sí terminar: ¿cómo se mezcla ese sentimiento social que no tiene nombre, que puede ser simplemente molestia, con un creciente discurso de derecho, específicamente con la noción de derechos económicos, sociales y culturales que establece que las personas no solamente son beneficiarias de planes sociales, sino que son titulares de derechos que pueden exigir prestaciones al Estado?

PM: Sí, pero tienes que tener cuidado con los conceptos de tu formación. Hay derechos y deberes, ¿cuáles son los deberes?

\section{CS: Esa es la parte que no se está cumpliendo...}

PM: No. Ese es un ángulo. Eso originó una de las discusiones en el Consejo y hay un debate al respecto de derechos y deberes. Pero lo que no queremos, que afecta a tu profesión, es judicializar el asunto. Resolvamos esas dos cuestiones para no caer en lo que se pone de moda.

CS: Los aumentos de los índices de judicialización de ciertos programas sociales van de la mano con la incapacidad del Gobierno, o del Estado, a responder a ciertas demandas sociales. Yo creo que uno es reflejo del otro, aunque comparto que no necesariamente es el medio más idóneo.

PM: Sí y no, porque es claro cómo puedes universalizar los derechos, como se ha dicho en el sector de salud a través del sistema de prestaciones mínimo del plan $\mathrm{AUGE}^{5}$. No obstante no opera de igual forma para todas las áreas, pues no es lo mismo cómo se universaliza en el sector educación o vivienda. En educación, por ejemplo, puede que destines igual cantidad de recursos para distintos grupos de estudiantes, pero que al término de la educación media obtengan puntajes muy dispares en la PSU.

\section{CS: Cobertura universal no es igual a calidad.}

PM: Claro. En Estados Unidos hay un estudio muy interesante sobre educación que revela que las diferencias que se producen en los puntajes se explican no por las variables de lo que pasa en los colegios. En un año los niños pasan la mitad del tiempo en el colegio y la otra mitad en la casa. Según este estudio, el factor desequilibrante es lo que pasa en la casa. Entonces, caricaturizando, si acá no te sacas 700 puntos en la PSU, podrías exigir al Estado que te mantenga de por vida con un millón de pesos mensuales.

\section{CS: Definir cuál es el estándar de cumplimiento es una labor compleja.}

PM: Lo que quiero decir es que puede ser más viable en ciertas áreas que en otras. Lo que se hizo en salud no es tan extrapolable a otros sectores, como el de educación. En Educación puedes asignar mucho dinero, como decía el profesor inglés del que te hablé, y no lograr ningún impacto. Por eso hay que tener el cuidado de evitar hacer un mal uso de los indicadores. Ahora, nosotros propusimos que, así como hay indicadores de inflación a largo plazo, que haya también indicadores sociales a largo plazo, por ejemplo, con qué niveles de inequidad, o diferenciales, entre el quintil más rico y el quintil más pobre queremos que esta sociedad funcione en los próximos veinte años. Y eso se podría ir midiendo año a año, y se irían evaluando los gobiernos, no sólo según la tasa de crecimiento, sino también por los niveles de equidad alcanzados.

\footnotetext{
Sistema de Acceso Universal con Garantías Explícitas de Salud.
} 